\title{
La intervención institucional en las micro y pequeñas empresas de la industria del calzado de Guadalajara
}

\section{$\mathrm{I}$} ntroducción

En este documento se analiza la intervención de las instituciones de fomento y desarrollo en las micro y pequeñas ${ }^{1}$ empresas (mypes) del sector del calzado en la ciudad de Guadalajara $^{2}$, el territorio más representativo de Jalisco.

Las instituciones que se analizan participaron de las experiencias de agrupamientos empresariales que se impulsaron en la industria del calzado en el periodo 1997-2001, fenómeno que se analiza de acuerdo con las ideas de Berry (1997), quien señala que un agrupamiento empresarial no sólo involucra a los mecanismos de protección conjunta sino también a la sustentabilidad del entorno industrial e institucional en su conjunto y a la elasticidad basada en la flexibilidad para modificar las condiciones de trabajo.

Se analizan aquí los actores más representativos y significativos del contexto local (zona metropolitana de Guadalajara), donde han conformado una dinámica particular con respecto a la cadena productiva y el entorno institucional de la industria zapatera; se considera el trabajo de las instancias políticas estatales dotadas de competencia en materia de desarrollo local,

* Investigadora del Departamento de Estudios del Pacífico de la Universidad de Guadalajara. particularizando la intervención de las instituciones en la pequeña industria, como el gobierno de Jalisco, la Cámara de la Industria del Calzado del Estado de Jalisco (CICEJ), y la participación de las instituciones financieras, como la unión de crédito y el programa de financiamiento del gobierno del estado.

El papel de la intervención institucional

Un aspecto determinante de las instituciones, de acuerdo con Casalet (2000), no se refiere sólo a su existencia, sino también a la capacidad que éstas pueden desarrollar para interrelacionarse $\mathrm{y}$ renovar las relaciones del tejido productivo, posibilitando una compleja red de cooperación tecnológica, financiera, de capacitación, cuya interacción y continuidad podría consolidar un sistema de información más integrado. Un elemento para desarrollar la capacidad tecnológica es la estrecha vinculación entre las políticas y los incentivos a nivel macro y las instituciones cuya finalidad es propiciar el entorno favorable.

Contrario a lo anterior, y siguiendo a Dini y Katz (1997), es cuando las instituciones operan en forma inadecuada, es decir, con un patrón ineficiente de atención y sin definición de calidad, establecen un comportamiento burocrático, de oportunismo y 
falta de respuesta, generando en consecuencia un uso ineficaz de los recursos empleados.

La intervención institucional, desde esta perspectiva, es particularmente importante en la evolución en ciertas fases del proceso de evolución industrial, en donde las diferentes instituciones juegan un papel significativo, proactivo y, sobre todo, responsable en el desarrollo de la interacción efectiva, principalmente entre las mypes, como, por ejemplo, en el contexto de los agrupamientos empresariales, y con ello establecer una política industrial que generalmente debería estar diseñada para tomar ventaja de la cooperación interfirma y fomentarla en el caso de no haberla (Casalet, 1997).

La provisión de servicios, sobre una base grupal, usualmente tiene sentido (por la disminución de los costos) debido a que las empresas se comprometen en niveles relativamente altos de cooperación y hay un incentivo para aumentarlos en especial entre los agrupamientos nacientes para apoyarse en diferentes niveles, como las asociaciones locales, los bancos, las instituciones de educación, capacitación e innovación tecnológica, las organizaciones no gubernamentales y en el mejoramiento de los sistemas de información (Serrano, 2001).

\section{La industria organizada del sector del calzado}

El Instituto Nacional de Estadística, Geografía e Informática ${ }^{3}$ (INEGI) tiene registradas (dato de 1999) 7,696 unidades productoras de calzado en el país, las cuales emplean a 108,102 personas. De este total nacional, Guanajuato se ubica en primer lugar con 3,548 (46\%) unidades y emplea a 66,688 (62\%) personas. Jalisco, en segundo lugar, cuenta con 900 (12\%) unidades económicas en las que emplea a 15,472 (14\%) personas (ver cuadro 1).

Los productores zapateros están integrados por tres cámaras industriales principalmente, que conservan la misma estructura de acuerdo al número de fabricantes: la Cámara de la Industria del Calzado del Estado de Guanajuato (CICEG), la Cámara de la Industria del Calzado del Estado de Jalisco (CICEJ) y la Cámara Nacional de la Industria del Calzado (CANAICAL) los cuales conforman el resto de integrantes a nivel nacional, (CIATEG, et al., 1993: 4). Esta distribución, considerada en 1993, es la que conservó la industria hasta el 2001.

De los productores de calzado organizados en cámaras por estados, en 2001, Jalisco, ubicado por su volumen en segundo lugar nacional, cuenta con sólo 371 empresarios registrados como miembros activos, de los cuales $85 \%$ son fabricantes de calzado y $15 \%$ están inscritos como socios cooperadores que forman parte de la cadena productiva. De ese $85 \%, 62 \%$ (195 empresas) en promedio, son empresas micro; las pequeñas empresas representan $34 \%$ de productores (107 empresas), mientras que $3 \%$ (10 empresas) forman el grupo de las medianas $\mathrm{y}$, finalmente, apenas alcanzan las empresas grandes a representar $0.95 \%$ del total (3 empresas) de integrantes de la CICEJ.

En contraste con estos datos, los funcionarios de la CICEJ tienen conocimiento ${ }^{4}$ de la existencia de alrededor de 1100 productores de calzado, de los cuales 800 se ubican en la zona

\begin{tabular}{lrrrrl}
\multicolumn{1}{c}{ Lugar } & Cuadro 1 & \multicolumn{1}{c}{ Especialización } \\
\hline Nacional & Unidades económicas & \multicolumn{1}{c}{$\%$} & Empleos & \% & Calzado de piel \\
Guanajuato & 7,696 & 100 & 108,102 & 100 & Caballero y niños \\
Jalisco & 3,548 & 46.10 & 66,688 & 61.69 & Dama y niñas \\
Edo. de México & 900 & $\mathbf{1 1 . 6 9}$ & $\mathbf{1 5 , 4 7 2}$ & $\mathbf{1 4 . 3 1}$ & Dama \\
Distrito Federal & 406 & 5.3 & 5,918 & 5.5 & Sandalia \\
Nuevo León & 274 & 3.5 & 3,597 & 3.3 & Tenis \\
\hline
\end{tabular}

Fuente: Censo industrial. INEGI 1999. 
metropolitana de Guadalajara, es decir, dos terceras partes, pero que no están registrados. Ante este contexto de reducida afiliación a la CICEJ, las cámaras industriales distan de ser un actor social activo, elástico y sustentable para el desarrollo industrial de la localidad, a diferencia de otros países industrializados, donde las asociaciones empresariales tienen mayor relevancia porque ayudan a dirigir estratégicamente a la industria, así como a implantar las diversas políticas de reestructuración productiva de la localidad.

En este sentido, se requiere una organización sectorial e industrial que represente los intereses de los empresarios para que acudan de forma autogestiva y tengan una visión de cadena productiva, donde el criterio asociacionista es útil para competir en el mercado globalizado, sin la necesidad del registro obligatorio en las cámaras industriales que prevaleció en la ley hasta 1997.

\section{Instituciones de financiamiento}

Debido al panorama económico, es difícil que se otorguen apoyos financieros para las mypes; en el caso del calzado este problema se agudiza debido a que del total del crédito otorgado por la banca comercial y de desarrollo en 1999, esta industria contaba con el índice de cartera vencida más elevado del sector (42\%) según el estudio de Bancomer; en el informe del Grupo Financiero Bital (GFB) este sector no sólo posee el índice de cartera vencida más elevado de la industria manufacturera, sino también uno de los más altos de la economía (GFB, 1997:60). ${ }^{5}$ Esta situación, además de llevar a esta industria a enfrentar serios problemas en la reestructuración de los créditos contratados con anterioridad, ocasionó que la banca la descartara de la obtención de nuevos créditos (Bancomer, 1999; GFB, 1997).

Sin embargo, las pequeñas empresas organizadas en agrupamientos empresariales pueden tener mayores posibilidades de obtener un crédito, debido a que la responsabilidad conjunta entre un grupo de pequeñas empresas puede ser útil para reducir la reacción de aversión al riesgo por parte de las instituciones financieras.

Con respecto a la experiencia en México, López Espinosa (1995) señala que la mayoría de las empresas pequeñas y medianas desconocen los accesos al crédito formal; además no cuentan con el personal, tiempo, ni capacidad para llenar los requisitos bancarios y por sus características tienen dificultad para ofrecer las garantías requeridas por las instituciones.

Por otro lado, el mismo autor indica que las sucursales de algunos bancos o instituciones financieras que dirigen recursos para las mypes desconocen los procedimientos y apoyos para éstas, no cuentan con personal especializado para evaluar proyectos a este sector empresarial, y el manejo de los criterios se respalda en las garantías ofrecidas y no en la viabilidad crediticia, de donde resulta la poca utilización de apoyos y el retraso excesivo en el otorgamiento del crédito.

Como muestra, de acuerdo con Berry (1997) esto se puede ver reflejado en la reducción de las ofertas financieras para el sector industrial, no sólo en México, sino también en América Latina, debido a que el papel de la banca de desarrollo como fuente de financiamiento para las pequeñas empresas ha disminuido su participación como parte de las reformas financieras. De esta manera, en palabras de Rubén Díaz ${ }^{6}$, director de la CICEJ "las micro y pequeñas empresas han tenido que implementar alternativas de financiamiento propias a través de ampliar los plazos de pago de sus proveedores o de apoyar al consumidor también con plazos".

\section{Uniones de crédito}

Un modelo de financiamiento con asociacionismo, que tuvo gran auge desde finales de la década de 1980 y hasta la primera mitad de la de $1990^{7}$, consiste en otra forma de asociación empresarial a través de las uniones de crédito $^{8}$, con las que se pueden obtener beneficios como el aval para el crédito, la compra de insumos en mejores condiciones, apoyo para 
asesoría y capacitación (entre los servicios más relevantes), (Pomar y Rivera, 1998).

La unión de crédito garantiza el acceso a las fuentes de financiamiento de la banca de desarrollo a menor costo; con ello, pueden disminuir los requisitos de información o garantía que deben presentarse para evaluar las solicitudes de crédito. Por medio de la unión de crédito pueden efectuarse, a mejores costos, compras en común de materia prima, tener acceso a mercados que en forma individual no podrían abarcar y contratar servicios en común, lo que permitirá disminuir costos e incrementar calidad y competitividad.

Sin embargo, desde 1993 la única forma de uso que tienen los socios de la Unión de Crédito de la Industria del Calzado (UCIC) es el área de compras en común, también conocida como departamento de comercialización, el cual consiste en que los proveedores hagan distribuciones directas a la UCIC, de esa forma pagar de gran mayoreo a los distribuidores y tener margen a precio más bajo para aumentar las utilidades y operación a través de los usuarios de aquélla

La UCIC cuenta con 380 socios registrados, de los cuales 120 son los integrantes más activos, debido a que hacen uso del departamento de compras en común, "área que le da vida artificial a la institución" " pero no es normal porque sólo se hace uso de uno de los servicios de todos para los que fueron creadas estas instituciones, como se señala en la Ley. ${ }^{10}$

Se considera que todavía no se entiende por parte de los pequeños productores del calzado el beneficio de trabajar en forma colectiva e institucionalizada para realizar compras en común, puesto que el sentido de solidaridad, confianza y el interés de participar en este organismo no se percibe claramente, ya que del total de los productores registrados en la UCIC, menos de la tercera parte solicitan el servicio.

Para ser miembro de la unión es necesario aportar un capital, el cual la mayoría de las veces no se tiene, e impide formar parte de ella. Las recurrentes crisis económicas que se han vivido desde 1982 no han permitido que los empresarios se capitalicen; al contrario, se ha creado un proceso de descapitalización por la falta de planeación de los empresarios y el otorgamiento de recursos de la banca de desarrollo, sin prever y fomentar la responsabilidad del pago en las figuras financieras como la unión de crédito.

Los principales problemas que enfrentan las uniones de crédito son: operan con capital muy reducido, cuentan con personal no especializado para ofrecer servicios, el fondeo es limitado, tiene un alto índice de cartera vencida, y el marco legal es estricto y restringido porque exige un encaje legal, sin permitir captación (Pomar y Rivera, 1998:80).

\section{Financiamiento del gobierno del estado de Jalisco}

Para que el proceso productivo funcione adecuadamente se requiere de la participación e interrelación de diferentes instituciones, principalmente del manejo de una política industrial con intervención de un gobierno y un sistema financiero locales que tengan un elevado desarrollo en sus instituciones públicas, así como un sentido de negociación y consecución de acuerdos sociales a escala local por los actores sociales que lo integran (Alessandrini y Canullo, 1998).

Sin embargo, en palabras del diputado federal Jorge Urdapilleta Núñez, el papel del gobierno federal no ha sido eficiente en diseñar y poner en marcha políticas de apoyo para la pequeña y mediana industria, debido a que han operado 551 diferentes programas, de los cuales 151 dependen de aquél para atender las necesidades de este sector empresarial, y 400 corresponden al sistema estatal; en esos programas, de cada peso que se presupuesta para fines de beneficio de las micro, pequeñas y medianas empresas, en el mejor de los casos, les llegan 30 centavos, situación que refiere una amplia estructura burocrática, más que responder a sus objetivos (Fortoul, 2002:4). 
En el caso de Jalisco, los sectores estratégicos industriales que definió el gobierno a partir de 1995, por ser un estado multisectorial, se organizaron en siete grupos industriales: agroindustria, electrónicatelecomunicaciones-informática, autopartesmetalmecánica-automotriz, textil-confección, cuero-calzado-marroquinería, madera-mueblesdecoración y minería-joyería-artesanía.

De acuerdo con Fernando Jiméne ${ }^{11}$, director sectorial de la Secretaría de Promoción Económica (Seproe) del estado de Jalisco, ante esta estrategia industrial, el sector de la electrónica-telecomunicaciones-informática fue de los que recibieron mayor atención en cuanto a los objetivos de los programas de gobierno donde las pequeñas empresas de la industria del calzado se ubicaron en segundo lugar, después de la electrónica, a pesar de reconocer (el funcionario) que Jalisco cuenta aproximadamente con $98 \%$ de mypes y el reconocimiento de las potencialidades de las mypes; por ello interesó a esta entidad de gobierno conocer los procesos que experimentaron en forma exitosa países como Italia, donde se lleva a la práctica el modelo productivo compuesto por redes de competencia y cooperación, como el asociacionismo y los agrupamientos empresariales.

La política industrial que diseñó el gobierno estatal por medio de la Estructura del Plan Estatal de Desarrollo 1995-2001, promovió entre sus diferentes programas el de Agrupamientos Empresariales (Agrems), dirigido a las mypes a fin de poder intervenir en el asociacionismo entre ellas , y con el objeto de emular un modelo de productividad flexible para alcanzar el desarrollo y madurez de industrias de clase mundial e innovadoras (Cárdenas, 1999).

De la misma forma, el gobierno de Jalisco también creó, desde 1996, el Fondo Jalisco (Fojal), un programa de fomento empresarial para el desarrollo de la micro y pequeña industria a través de un plan de financiamiento local, el cual brinda, como característica especial, capacitación al adquirir los recursos; con ello apenas se percibe un cambio de actitud en los programas gubernamentales hacia esta área, al tener que recibir un curso para saber administrar el financiamiento que ofrecen. Lo anterior presenta un escenario novedoso en el que cada vez es más importante el uso y manejo de programas de fuentes financieras con sistemas de capacitación para el aprovechamiento y retorno de los préstamos.

La oferta financiera del programa de Fondo Jalisco consiste en un monto promedio por empresa, pero es una cantidad que, de acuerdo a la opinión del director de la CICEJ, no alcanza para la adquisición de una máquina o para innovación tecnológica, de manera que son recursos rezagados que no se han otorgado a esta industria tradicional, considerada como de las menos viables para aprobar un estudio de crédito, debido a que se otorgaron 90 en promedio por año, para un total de 1100 empresas de la misma. Así se ve que es mínimo el apoyo, sobre todo por el monto.

Por ello, la creación y el fomento de esquemas de asociacionismo entre las empresas micro, pequeñas y medianas ayuda a tener acceso a fuentes internacionales de financiamiento más competitivas, puesto que la banca de desarrollo local de apoyo a la exportación ofrece una tasa de interés poco competitiva, superior al 200 ó $500 \%$, más de lo que se consigue en otros países, según la versión del asesor de Agrems de la CICEJ ${ }^{12}$, quien señaló que no existe una cultura de alianzas entre instituciones para competir, lo cual se traduce en una falta de competitividad institucional en el caso de los empresarios del calzado.

No obstante, todavía se percibe la falta de cultura financiera por parte de las empresas, así como de las instituciones de crédito, ya que es mínimo el conocimiento que tienen una de la otra; así, el proceso financiero congruente con las condiciones de los industriales y los bancos, desconocen los problemas y requerimientos industriales para la competitividad. 


\section{Comentarios finales}

El presente trabajo refleja que la interrelación de las diferentes instituciones dentro de la localidad analizada (gobierno, empresarios organizados en cámaras empresariales, así como organizaciones financieras) forman parte de la intervención interinstitucional. Se revisó el papel que desempeña cada una en Guadalajara para considerar su importancia en el proceso productivo de la micro y pequeña industria del calzado.

En Guadalajara, uno de tantos casos en México, no se contaba con una red institucional de apoyo a las pequeñas empresas que permitiera modificar sus condiciones de trabajo, debido al tardío reconocimiento del potencial de este sector y el tipo de empresas. Sin embargo, es hasta 1997 que se inician los apoyos más significativos a los encadenamientos productivos y la cooperación entre empresas.

Como se mencionó, dentro de los objetivos del gobierno estatal se otorgó mayor atención a la industria electrónica que a las industrias tradicionales, compuestas en su mayoría de pequeños establecimientos, como la del calzado. Al mismo tiempo, hasta ahora el gobierno se da cuenta de que este estrato productivo es competitivo no obstante las dificultades en la economía; una industria que sobrevive a pesar de las recurrentes crisis que se han generado desde 1982.

La revisión que se hizo de las diferentes instituciones, en el caso de Guadalajara, resalta la existencia de una red importante de apoyos institucionales para las mypes, pero todavía no se habla de una red sólida como lo define Casalet (2000:324): "representan algo más que una forma híbrida, ya que proveen una forma específica de coordinación que puede resolver problemas que los mercados ni las organizaciones afrontan"; se trata de apoyos aislados que inician en los agrupamientos empresariales y la cooperación entre empresarios, a los cuales todavía no se les puede llamar red articulada, pero que cuentan con el potencial para serlo. Para ello, todavía se presentan, de manera significativa, limitaciones de información y difusión efectiva de los recursos y programas, aspectos que dificultan la consolidación de relaciones más complejas a las que se puede agregar la débil vinculación entre la investigación, la formación técnica y la actividad industrial.

Las relaciones interinstitucionales con las pymes son una práctica que apenas se inicia puesto que todavía prevalece cierta desconfianza, a la que se debe afrontar urgentemente en las instituciones del gobierno estatal, en gremios organizados, así como en las universidades, para responder a las carencias que tiene la sociedad y no haya comportamientos inadecuados, reaccionando de acuerdo con el modelo de cooperación de $\operatorname{clan}^{13}$, en forma vertical, horizontal y diagonal, es decir, con un concepto mixto acorde a los requerimientos de la pequeña industria.

\section{Bibliografía}

Alessandrini P. y Giuseppe Canullo, "Los distritos industriales de Las Marcas, Italia: evolución y perspectivas", en Carlos Alba et al. (comps.), Las regiones ante la globalización, El Colegio de México - CEMCA ORSTOM, México, 1998, pp. 591-611.

Bancomer, Reporte anual, riesgos sectoriales Bancomer, fabricación del calzado, México, 1999.

Berry R. Albert, Análisis de la «mejor práctica» en la cooperación interempresarial. Pensar globalmente y actuar regionalmente, hacia un nuevo paradigma industrial para el siglo XXI, FCE, 1997, México, pp. 229-291.

Boston Consulting Group LTD y Grupo Consultor Ejecutivo, "Industria del Calzado", Bancomext, SECOFI, México, 1988.

Cárdenas, Jiménez, Alberto, $4^{\circ}$ Informe de Gobierno del Estado de Jalisco, 1999.

Casalet, Mónica, "La cooperación interempresarial: una opción para la política industrial", Comercio Exterior, México, vol. 47, núm.1, 1997, pp. 8-15.

"Éxitos y desaciertos en la cooperación interempresarial: un análisis de dos experiencias, Chile y Argentina", en Isabel Rueda Peiro y Nadima Simón Domínguez (coords.), Asociación y cooperación de las micro, pequeña y mediana empresas: México, Chile, Argentina, Brasil, Italia y España, PorrúaUNAM, México, 1999. construcción del entorno: nuevas instituciones e identidades", en Enrique de la Garza Toledo (coord.), Tratado latinoamericano de sociología del trabajo, COLMES-FLACSO-UAM-FCE, México, 2000, pp. 312-341. CEO-CICEJ/ Centro de Estudios de Opinión de la 
Universidad de Guadalajara y Cámara de la Industria del Calzado en Jalisco, Estudio de mercado del calzado para dama Guadalajara, 1997.

CIATEG, CICEG, CEESP/ Centro de Investigación y Asistencia Tecnológica del Estado de Guanajuato, Cámara de la Industria del Calzado del Estado de Guanajuato, Centro de Estudios Económicos del Sector Privado, Información estadística del sector calzado, s.e., México, 1993.

Coordinadora Sectorial (COSEC), Plan estratégico de desarrollo. Sector cuero, calzado, proveeduría, s.e., México, 1995.

Dini, M. y J. Katz, "Nuevas formas de encarar las políticas tecnológicas. El caso de Chile”, Comercio Exterior, México, vol. 47, núm. 8, 1997.

Fortoul, Sol, Público, Guadalajara, 27 de abril de 2002, p.4.

Grupo Financiero Bital (GFB), Anuario 1997, "Evaluación de riesgo y perspectivas", Dirección de análisis y estudios económicos, México, 1997.

Instituto Nacional de Estadística Geografía e Informática, (INEGI), Censo Económico 1999.

López Espinosa, Mario, "Consideraciones sobre el acceso de la pequeña empresa y el taller artesanal a los mecanismos formales de financiamiento", en Thomas Calvo y Bernardo Méndez Lugo (coords.), Micro y pequeña empresa en México frente a los retos de la globalización, CEMCA-Misceláneas, México, 1995, pp. 71-94.

Pomar Fernández, Silvia y Martín Rivera Guerrero, Alternativas de financiamiento para la micro, pequeña $y$ mediana empresa, UNAM, México, 1998.

Saraví, Gonzalo, «Relaciones de cooperación interfirmas», en Cultura empresarial en un distrito industrial mexicano: comunidad y relaciones interfirmas, Los estudios de asociación en América Latina, Chicago, septiembre, 1998, pp. 1-14.

Serrano, Diana, "Las estrategias de cooperación de los micro y pequeños empresarios del calzado de Guadalajara (1986-2000), tesis de maestría, Universidad de Guadalajara, 2001.

\section{Notas}

1 En México, de acuerdo con el Diario Oficial de la Federación del 30 de marzo de 1999, la estratificación empresarial se compone, según el número de empleados, de la siguiente manera:

a) Sector industrial: la micro empresa de 0 a 30 trabajadores, la pequeña de 31 a 100 , la mediana de 101 a 500 y la grande de 501 en adelante.

b) Sector comercio: microempresa de 0-5 trabajadores, pequeña empresa de 6-20 trabajadores, mediana empresa de 21-100 trabajadores y gran empresa de 101 en adelante.

c) Sector servicios: microempresa de 0-20 trabajadores, pequeña empresa 21-50 trabajadores, mediana empresa de 51-100 trabajadores y gran empresa de 101 en adelante.

2 Este trabajo forma parte del trabajo de investigación de mi tesis de maestría.

3 INEGI. Censo industrial 1999.
4 Esta información la proporcionó el gerente administrativo de la CICEJ en una entrevista realizada el 25 de abril del 2000.

5 "El panorama crediticio del sector en 1996 mantenía $0.4 \%$ del total de crédito otorgado por la banca comercial y de desarrollo, este dato con respecto al de 1995, la cartera total se incrementó $52 \%$, mientras que la vencida lo hizo en $42 \%$ "(sic).

6 Información obtenida en una entrevista realizada el 25 de abril de 2000.

7 Para ilustrar la experiencia de operación de estos organismos financieros en el sexenio de 1988-1994, de 63 uniones de crédito que había en el país en 1989, llegaron a ser 527 en 1994 (Proceso, núm. 1222, abril 2, 2000).

8 La Ley General de Organizaciones y Actividades Auxiliares de Crédito, y los artículos 39, 40 y 41 consideran a las uniones de crédito como organizaciones auxiliares de crédito, constituidas como sociedades anónimas de capital variable que agrupan a personas físicas y morales micro, pequeñas y medianas, aglutinadas por sus áreas productivas o actividades como agropecuarias, comerciales e industriales o mixtas, que por medio de la cooperación buscan resolver necesidades financieras en forma más directa y oportuna, debido a que tienen mayor acercamiento al sistema financiero, así como otros beneficios derivados de las actividades que realizan en común, como abasto, comercialización y asistencia técnica.

9 Señalamiento que proporcionó el director de la Unión de Crédito de la Industria del Calzado (UCIC).

10 Información obtenida en una entrevista con el director de la UCIC, mayo 2000.

11 Información obtenida a través de una entrevista realizada en octubre de 2000 .

12 Información obtenida a través de una entrevista realizada en mayo de 2000 .

13 De acuerdo con Saraví (1998:6) la cooperación de clan consiste en formas de relación más novedosas, en proceso de formación, que intentan una mayor integración de sus recursos para el logro de objetivos comunes que no comprometen la autonomía individual, pero ayudan a reforzarla, de acuerdo con niveles verticales, horizontales y mixtos, donde cada nivel incrementa el riesgo y el compromiso del objetivo de cooperación $\mathrm{m!:}$ 